\title{
Performance Analysis of Dynamic Wavelength Allocation Algorithm for TWDM PON with Inexpensive ONUs
}

\author{
Man Soo Han \\ Department of Information and Communications Eng., Mokpo National University \\ Jeonnam, Republic of Korea \\ mshan@mokpo.ac.kr
}

\begin{abstract}
In this paper, we introduce a new dynamic wavelength allocation algorithm for a TWDM PON (time and wavelength division multiplexed passive optical network) having inexpensive ONUs (optical network units). The inexpensive ONU has a cheap and lowspeed wavelength tuner. The cheap tuner takes from few milliseconds to few seconds to change its wavelength. Most dynamic wavelength allocation algorithms assume that an ONU has a perfect tuner whose tuning time is zero. Unlike most existing algorithms, the proposed algorithm does not ignore the tuning time of an ONU tuner. In the proposed algorithm, an OLT (optical line termination) uses an average grant amount to predict the average packet arrival rates of all ONUs. The OLT decreases the number of active upstream wavelengths if the average grant amount is less than the bandwidth amount of the upstream wavelengths. Otherwise, the OLT increases the number of active upstream wavelengths. Using computer simulations, we show performance of the proposed algorithm and the average number of active upstream wavelengths.
\end{abstract}

Keywords: Dynamic wavelength allocation, TWDM PON, low-speed tuner, dynamic bandwidth allocation, performance evaluation

\section{Introduction}

TWDM PON (time and wavelength division multiplexed passive optical network) consists of an OLT (optical line termination) and multiple ONUs (optical network units). TWDM PON is the core technology of the NG-PON2 (next-generation passive optical network stage 2). Unlike an XG-PON (10-Gigabit-Capable passive optical network) which uses a single upstream wavelength and a single downstream wavelength, TWDM PON uses multiple upstream wavelengths and multiple downstream wavelengths. TWDM PON can vary the number of active wavelengths. Also TWDM PON can change the upstream and downstream wavelengths of an ONU [1-4, 8].

In TWDM PON, since a broadcasting mechanism is used to send packets from an OLT to ONUs, how the downstream wavelengths are used is not crucial for performance. However, since the TDM (time division multiplexing) mechanism is used to send packets from ONUs to an OLT, how the up-stream wavelengths are used is crucial in performance. An ONU requires a time to change its upstream wavelength [9]. Most researches assumed that the wavelength switching time of a tuner is zero or negligible [5]. The tuner price is high if its tuning time is very small so that the ONU price will be increased. If an ONU uses a low-speed tuner, its price will be decreased but the ONU cannot change its wavelength in real time. In addition, in [5], multiple ONUs can simultaneously change their upstream wavelengths at a time. However, only a single ONU can change its upstream wavelength at a time in the TWDM PON technology [6]. In this paper, we study performance of a new dynamic wavelength allocation algorithm when the low-speed tuner is used in an ONU and when only one ONU can change its upstream wavelength at a time. 
In [5], the number of active upstream wavelengths was determined by using the request sum of ONUs under the assumption that the tuning time is zero. The number of active upstream wavelengths is determined in a way that the bandwidth sum of upstream wavelengths is greater than or equal to the sum of requests. When the tuning time is not zero, however, we cannot use the request sum for the calculation of the number of upstream wavelengths. The reason is that the request information includes not only the newly arrived packets but also the packets arrived earlier but have not been serviced. The average request is not equal to the average packet arrival rate. In addition, in [5], it was assumed that ONUs simultaneously can change their upstream wavelengths.

In [4], a new dynamic wavelength allocation algorithm was introduced for a TWDM system in which an ONU has a non-zero tuning time and only a single ONU can change its upstream wavelength at a time. In [4], the proposed algorithm uses grant information instead of request information to calculate the number of upstream wavelengths. The OLT obtains an average grant amount during a predetermined period. If the average grant amount is far less than the maximum upstream bandwidth, it means the packet arrival rate is far less than the maximum upstream bandwidth. In this case, the OLT decreases the number of upstream wavelengths. If the average grant amount is almost equal to the maximum upstream band-width, it implies that the packet arrival rate maybe greater than the maximum upstream bandwidth. In this case, the OLT increases the number of upstream wavelengths. However, performance of the proposed algorithm was not shown in [4].

In this paper, we explain the algorithm of [4] and then show performance of the algorithm of [4]. Using computer simulations, we evaluate performance of the proposed algorithm under the self-similar traffic. We vary the input load rates of ONUs and then show the proposed algorithm effectively changes its number of active upstream wavelengths. We compare the proposed algorithm with the dynamic wavelength and bandwidth allocation for QoS (DAQ) algorithm that fully uses the all upstream wavelengths [5]. We show that performance of the proposed algorithm is comparable to that of DAQ.

\section{Dynamic Wavelength Allocation}

In this paper, we assume that a TWDM PON has four upstream wavelengths and four downstream wavelengths. We represent the four upstream wavelengths as $\lambda_{1}$, $\lambda_{2}, \lambda_{3}$, and $\lambda_{4}$. Also we denote the four downstream wavelengths as $\lambda_{5}, \lambda_{6}, \lambda_{7}$, and $\lambda_{8}$. Each ONU can use only one of the upstream wavelengths when it transmits packets to the OLT. Each ONU can use only one of the downstream wavelengths when it receives packets from the OLT. We suppose that the bandwidth of each wavelength is $2.5 \mathrm{Gbps}$ in this paper. Figure 1 depicts a TWDM PON system.

Let request (j) and grant $(j)$ be the request amount and the grant amount of the queue $\mathrm{j}$, respectively. The grant amount grant (j) is determined by the OLT based on the request amount request (j). When the tuning time of an ONU tuner is zero, the number of active upstream wavelengths can be immediately calculated from the sum of request $(\mathrm{j})$.

If the tuning time of an ONU tuner is not zero, we cannot calculate the number of active upstream wavelengths from the sum of request $(\mathrm{j})$. The reason is an elapse time between the time that the sum of request (j) is calculated and the time that upstream wavelength is changed. Since every operation of a TWDM PON is synchronous with an FD (frame duration) whose length is fixed to $125 \mu \mathrm{s}$, the sum of request (j) is calculated in each FD. It is well known that the wavelength change takes from few milliseconds to few seconds. Therefore, the sum of request $(j)$ at the current FD is different from the sum of request $(j)$ at the FD that wavelength is changed. 


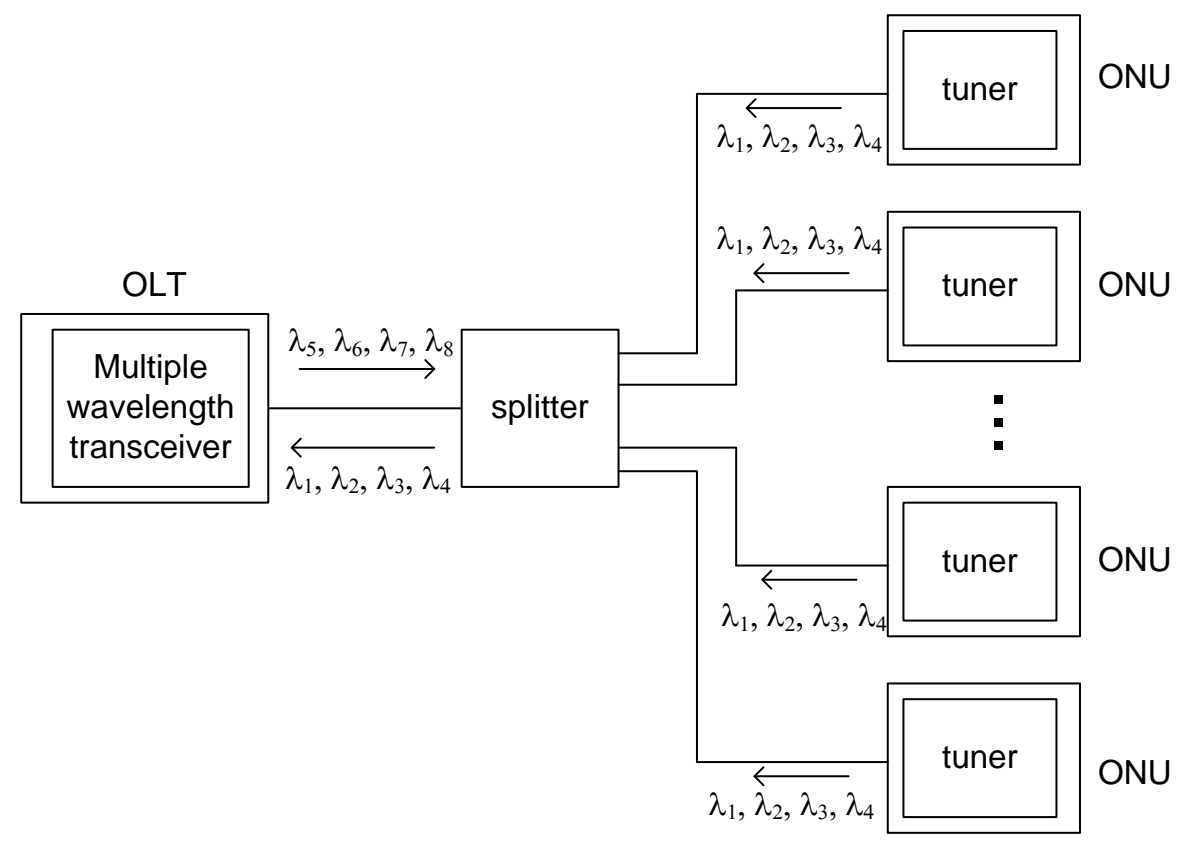

Figure 1. TWDM PON System

Since the tuning time is not zero, one possible solution for a dynamic wa velength allocation is using an average packet arrival rate. The number of active upstream wavelengths can be easily determined if we know the average packet arrival rate. It should be mentioned that we cannot use the average value of request (j) to calculate the average packet arrival rate. Let arrival $(j)$ be the packet arrival rate of queue $j$ during an FD. Since request (j) includes not only the newly arrived packets but also the packets arrived previous FDs and have not been serviced, the average value of request $(j)$ does not provide the information of the average packet arrival rate.

For example, consider two cases that find an average packet arrival rate over a time duration $T$. The first case is that the initial value of request $(j)$ at the beginning of $\mathrm{T}$ is 100 bytes and we have arrival $(\mathrm{j})=0$ and grant $(\mathrm{j})=0$ during $\mathrm{T}$ FDs. Then the sum of request $(\mathrm{j})$ during T FDs is 100 bytes. The second case is that the initial value of request $(j)=0$ at the beginning of $T$ FDs, and we have arrival $(j)=100$ bytes and grant $(j)=0$ during T FDs. Also we get the sum of request $(j)=100$ bytes. The average values of request ( $j$ ) are equal in the two cases. But the arrival ( $j$ ) is different in the two cases.

In this paper, we propose a new method to estimate the average packet arrival rate using grant ( $\mathrm{j}$ ) instead of using request $(\mathrm{j})$ and arrival( $\mathrm{j})$. Let a variable $A$ be the sum of grant $(j)$ for all queues. That is

$$
A=\sum_{j} \operatorname{grant}(j) .
$$

In addition, suppose a variable $\mathrm{G}$ is the average value of $\mathrm{A}$ over $\mathrm{T}$ FDs. Also let a variable $\mathrm{C}$ is the maximum bandwidth sum of the upstream wavelengths that are used in T FDs. Then we have $\mathrm{C}=\mathrm{n} \times 2.5 \mathrm{Gbps}$ where $\mathrm{n}$ is the number of the active upstream wavelengths during T FDs.

If $\mathrm{G}<<\mathrm{C}$ then it is clear that the maximum bandwidth of upstream wavelengths was larger than the average packet arrival amount during T FDs. It means the average packet arrival rate was the same as the average grant rate. Therefore in this case, the active number of upstream wavelengths for the next T FDs is obtained by

$$
n=\lceil G / 2.5 G b p s] \text {. }
$$


If we have $\mathrm{G} \approx \mathrm{C}$ then it means that the maximum bandwidth sum of upstream wavelengths might be less than the average packet arrival amount during T FDs. In this case, we cannot find the exact difference between the packet arrival amount and the maximum bandwidth. Therefore, it is unclear how much we need to increase the number of active upstream wavelengths in the next T FDs. In this paper, we propose two methods to solve this problem. The first method is based on the worst case that the average packet arrival rates of all ONUs were maximum during T FDs. In this case, we have to use all upstream wavelengths. That is $n=4$ at the next T FDs.

The second method is based on the optimistic case that the average packet arrival amount is slightly greater than the maximum bandwidth sum of upstream wavelengths during T FDs. We assume the difference between the packet arrival amount and the maximum upstream bandwidth is less than the maximum bandwidth of a single wavelength. Therefore, we need one more upstream wavelength at the next $\mathrm{T}$ FDs. That is $\mathrm{n}=\mathrm{n}+1$ at the next T FDs.

The first method has an advantage in good performance and has a disadvantage in power consumption since all upstream wavelengths are used in the next T FDs. The second method has an advantage in the power consumption since it minimizes the number of the active upstream wavelengths. But the second method has a disadvantage in performance in case the average packet arrival amount is greater than the bandwidth of the active upstream wavelengths at the next T FDs.

We show the pseudo codes for the first and second methods in Figures 2 and 3, respectively. The variable $\alpha$ is a proportional coefficient which is greater than 0 and less than 1 .

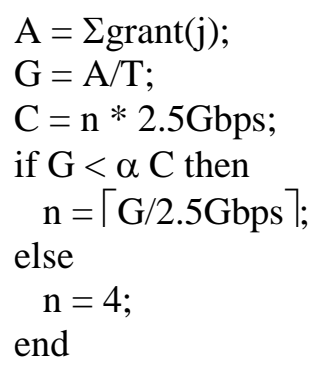

Figure 2. Pseudo Code of the First Method

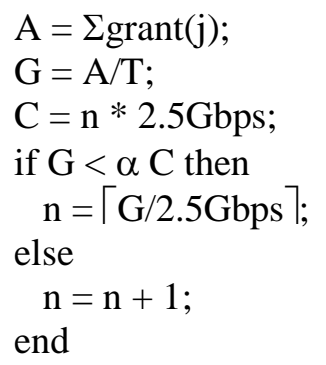

Figure 3. Pseudo Code of the Second Method

\section{Performance Evaluation}

We consider a TWDM PON system with 32 ONUs and 4 upstream wavelengths. The bandwidth of each upstream wavelength is 2.5 Gbps. The maximum input bandwidth of each ONU user interface is $400 \mathrm{Mbps}$. Also each ONU has three queues for T-CONT (transmission container) types 2, 3 and 4, respectively. In the TWDM PON technology, a service class is known as a T-CONT type. The size of each queue is 10 Mbytes. We use the scheduling algorithm of [5] for DBA (dynamic bandwidth allocation) operation. In [5], a dynamic wavelength and bandwidth 
allocation for QoS (DAQ) algorithm was introduced that fully uses the all upstream wavelengths. In [5], the tuning time for an upstream wavelength of an ONU is zero and multiple ONUs can simultaneously change their upstream wavelengths. We compare the second proposed method with the DAQ. Since one of the main goals of the dynamic bandwidth allocation is the power saving, we only consider the second method in this performance evaluation.

As in [5], we first reserve a service bandwidth for each queue. Let queue(j) be the queue $j$. Let $A(j)$ be the service amount of queue(j) during $S(j)$ which is the service interval of queue(j). For queue(j) of the T-CONT type 2, we have $A(j)=15,624, S(j)$ $=5$, which is equivalent to $200 \mathrm{Mbps}$. For each queue(j) of the T-CONT types 3 and 4 , we set $A(j)=31,248$, and $S(j)=10$, which is equivalent to $200 \mathrm{Mbps}$. We assume the variable $\alpha$ is 0.8 .

We use the self-similar traffic model for the input traffic of an ONU [7]. The traffic model is generated by a number of Pareto distributed on-off processes whose shape parameters are given by 1.2 and 1.4 , respectively. The packet size follows the tri-modal distribution that the packet sizes are 64, 500 and 1500 bytes with the fractions of $60 \%, 20 \%$ and $20 \%$, respectively. The load fractions of T-CONT types 2,3 , and 4 of an ONU are $\rho / 3, \rho / 3$, and $\rho / 3$, respectively, where $\rho$ is the input load rate of a single $\mathrm{ONU}$.

\subsection{Case 1}

The input load rate $\rho$ varies from $\lambda / 2$ to $\lambda$ with the changing interval $L$ as shown in Figure 4. In this simulation, we set $\mathrm{L}=5000$ frame durations. The load pattern is repeated for every $5 \mathrm{~L}$. In this simulation, we increase $\lambda$ from 0.1 to 0.99 to evaluate performance of the proposed method. For the proposed method, we assume the tuning time of an ONU is 10 FDs. Also according to the G.989.3 recommendation [6], only a single ONU can change its upstream wavelength at a time. This limitation is applied to the proposed method too.

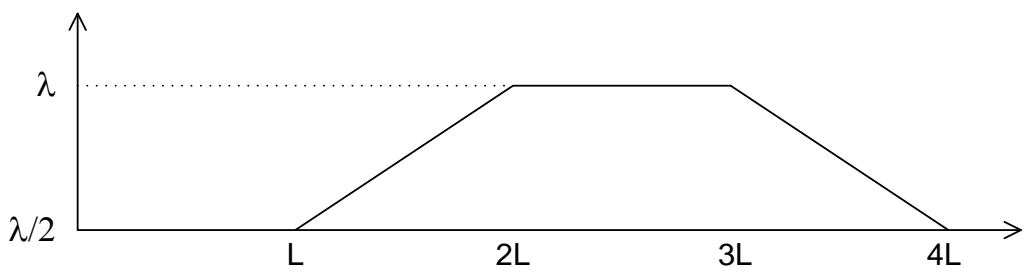

Figure 4. Input Load Rate

Figure 5 shows the mean packet delays of T-CONT types 2 and 3. Figure 6 illustrates the mean packet delays of T-CONT type 4 for the proposed method and the DAQ. As we can see performance of the proposed method is comparable to the DAQ for T-CONT types 2 and 3 despite many realistic limitations in the proposed method.

The performance difference of Figure 6 is explained by Figure 7 that shows the average number of the active upstream wavelengths during simulation. Since the DAQ allocates the upstream wavelengths for load balancing of all upstream wavelengths and the proposed method allocates the upstream wavelengths based on the grant amount, the average number of the active upstream wavelengths of the DAQ is greater than that of the proposed method. 


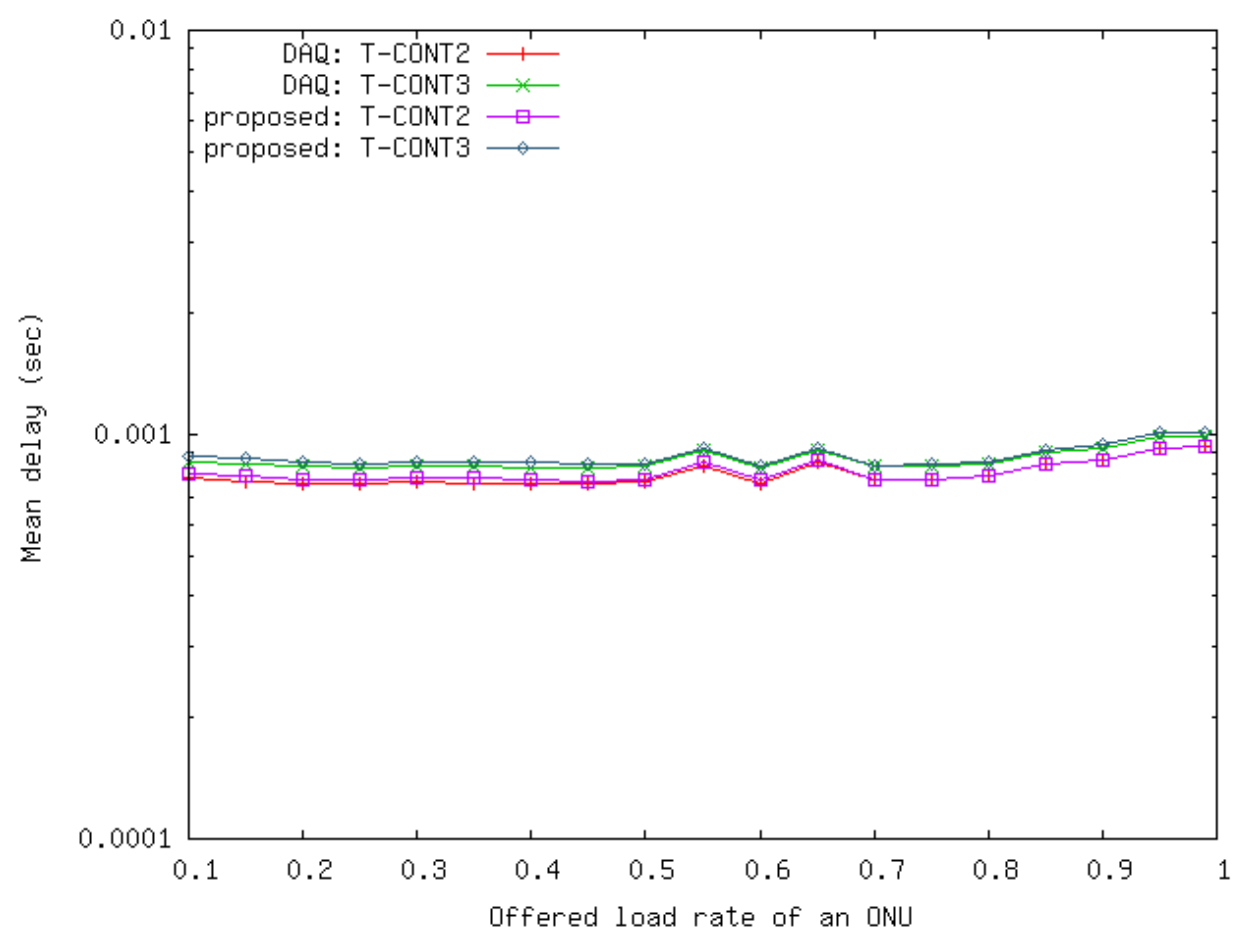

Figure 5. Mean Packet Delays of T-CONT Types 2 and 3

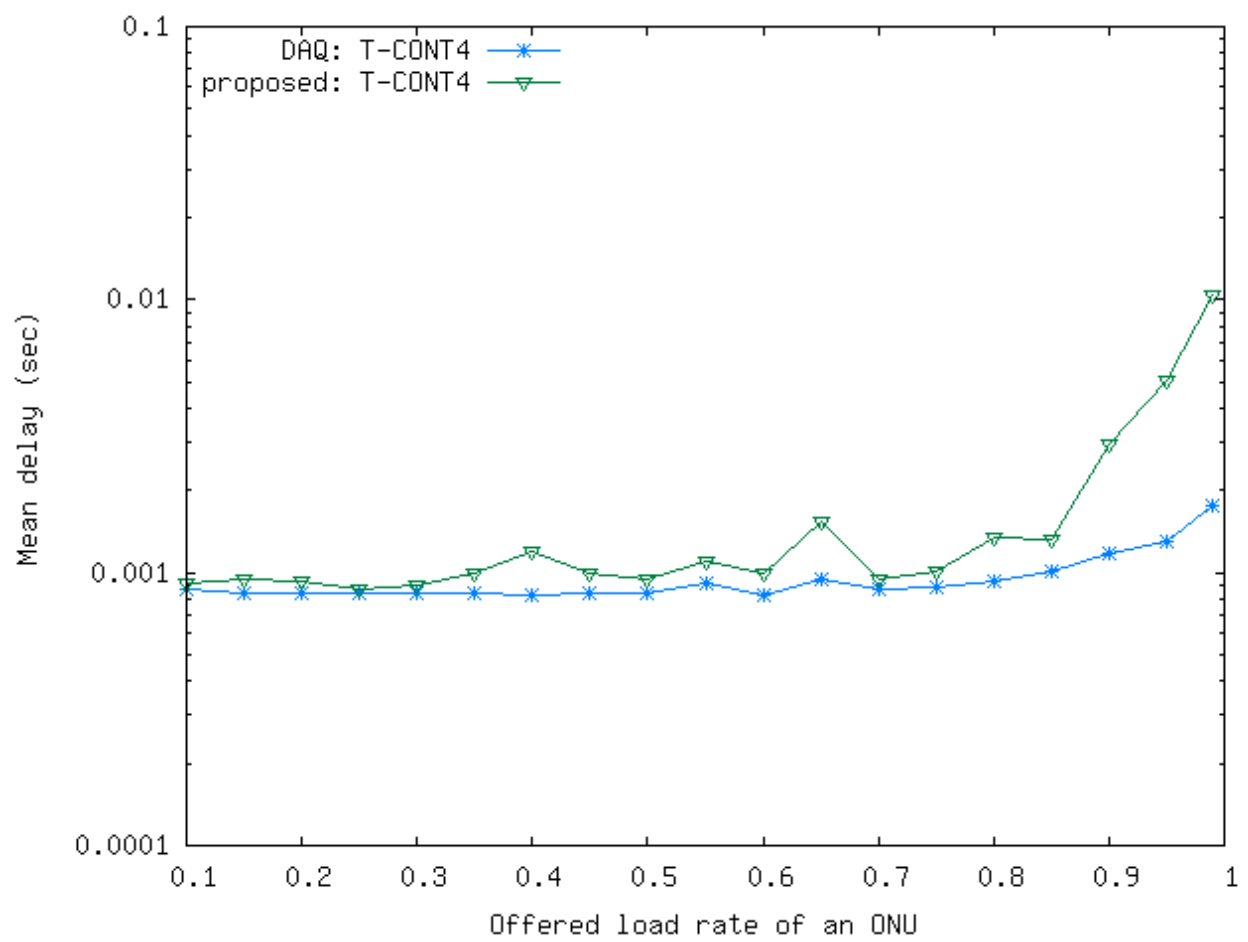

Figure 6. Mean Packet Delays of T-CONT type 4 


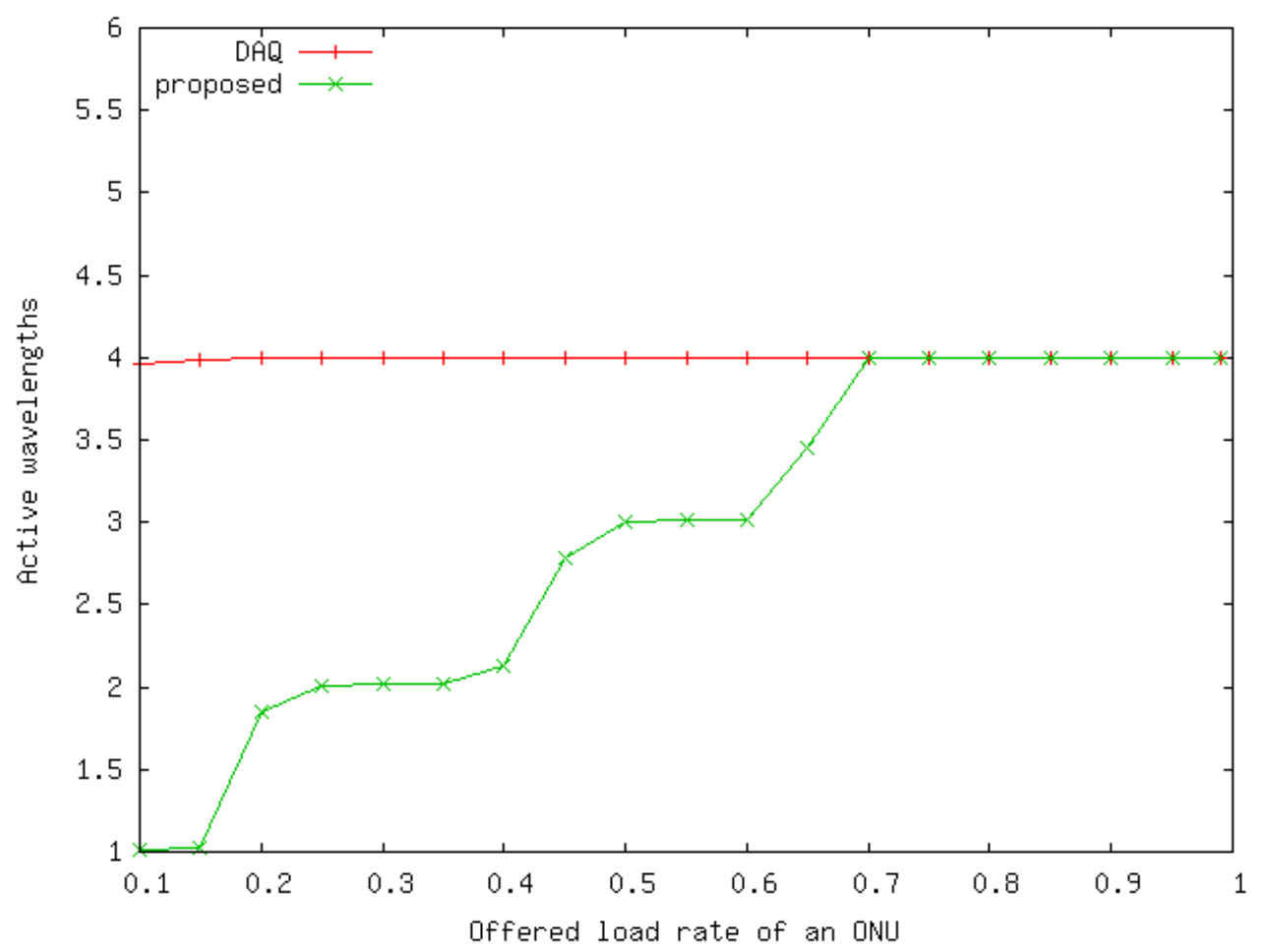

Figure 7. Average Number of Active Upstream Wavelengths

\subsection{Case 2}

Now we vary the input load rate $\rho$ from $\lambda / 4$ to $\lambda$ with the changing interval $L$ as shown in Figure 8. Also, we set $\mathrm{L}=7000$ frame durations and the load pattern is repeated for every $5 \mathrm{~L}$. Other parameters are equal to those of the case 1.

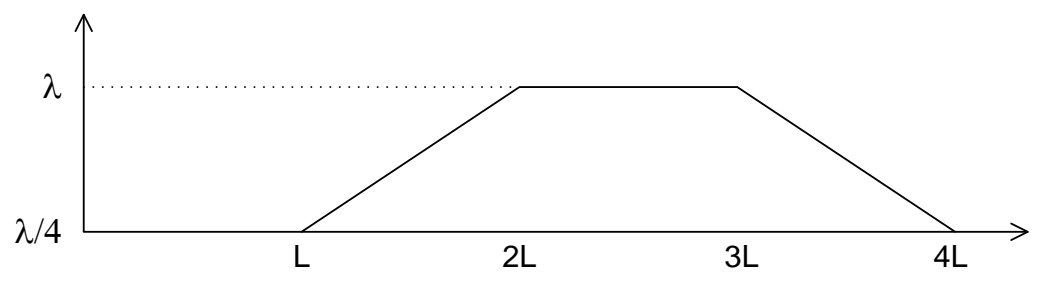

Figure 8. Input Load Rate

Figure 9 depicts the mean packet delays of T-CONT types 2 and 3. Figure 10 shows the mean packet delays of T-CONT type 4 for the proposed method and the DAQ. Also, performance of the proposed method is comparable to the DAQ for T-CONT types 2 and 3 despite that many realistic limitations are considered in the proposed method. Figure 11 shows the average number of the active upstream wavelengths during simulation for each algorithm. 


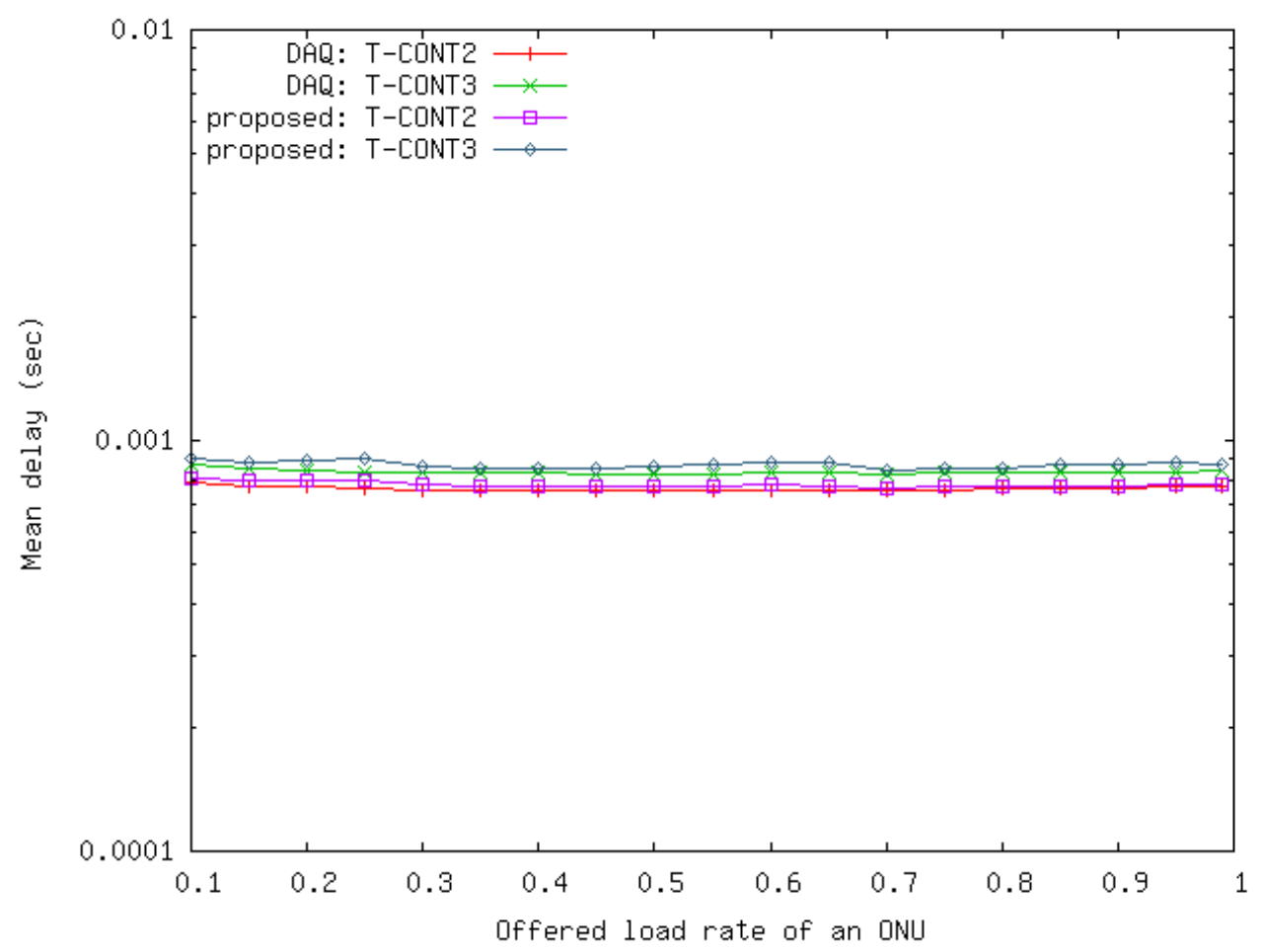

Figure 9. Mean Packet Delays of T-CONT Types 2 and 3

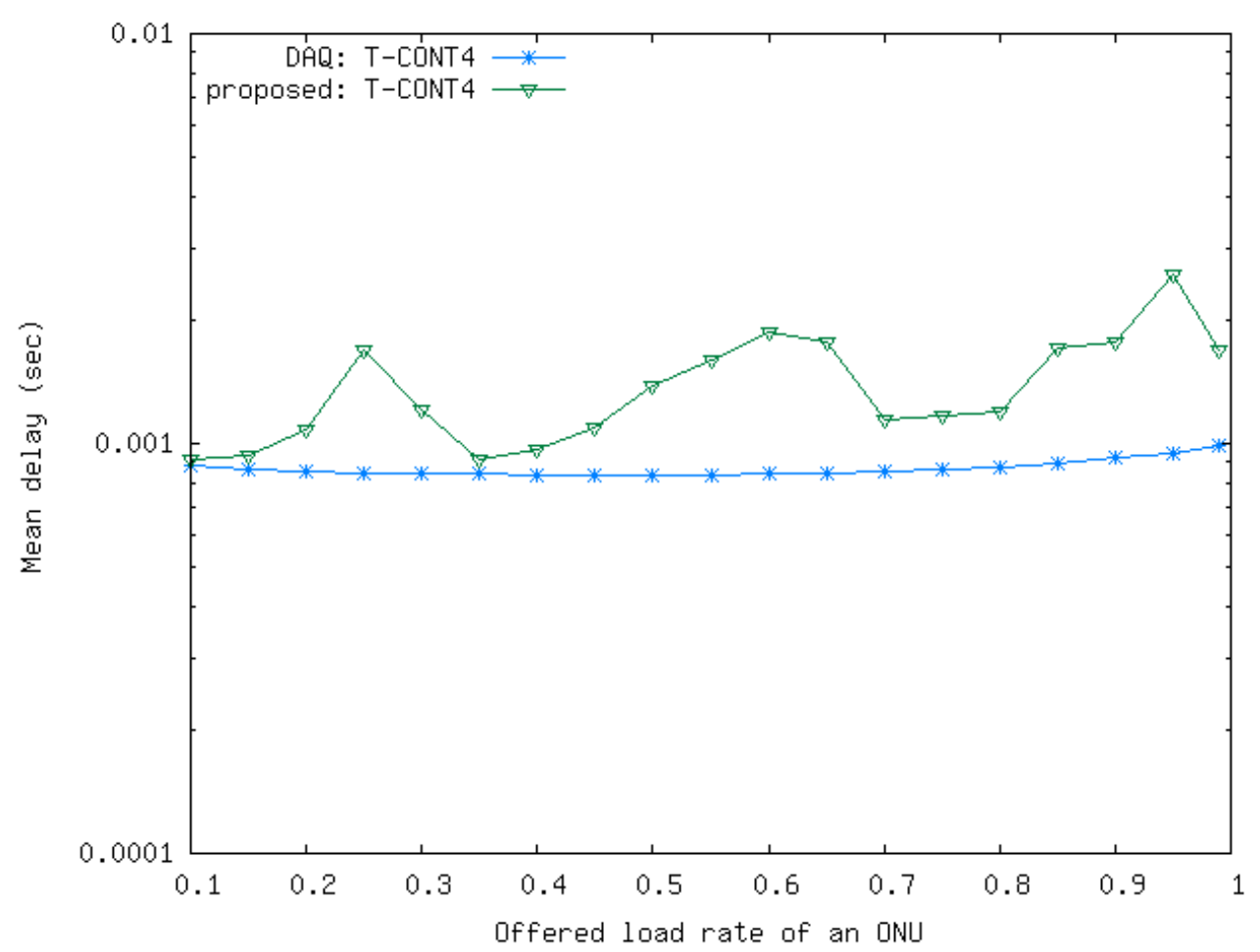

Figure 10. Mean Packet Delays of T-CONT Type 4 


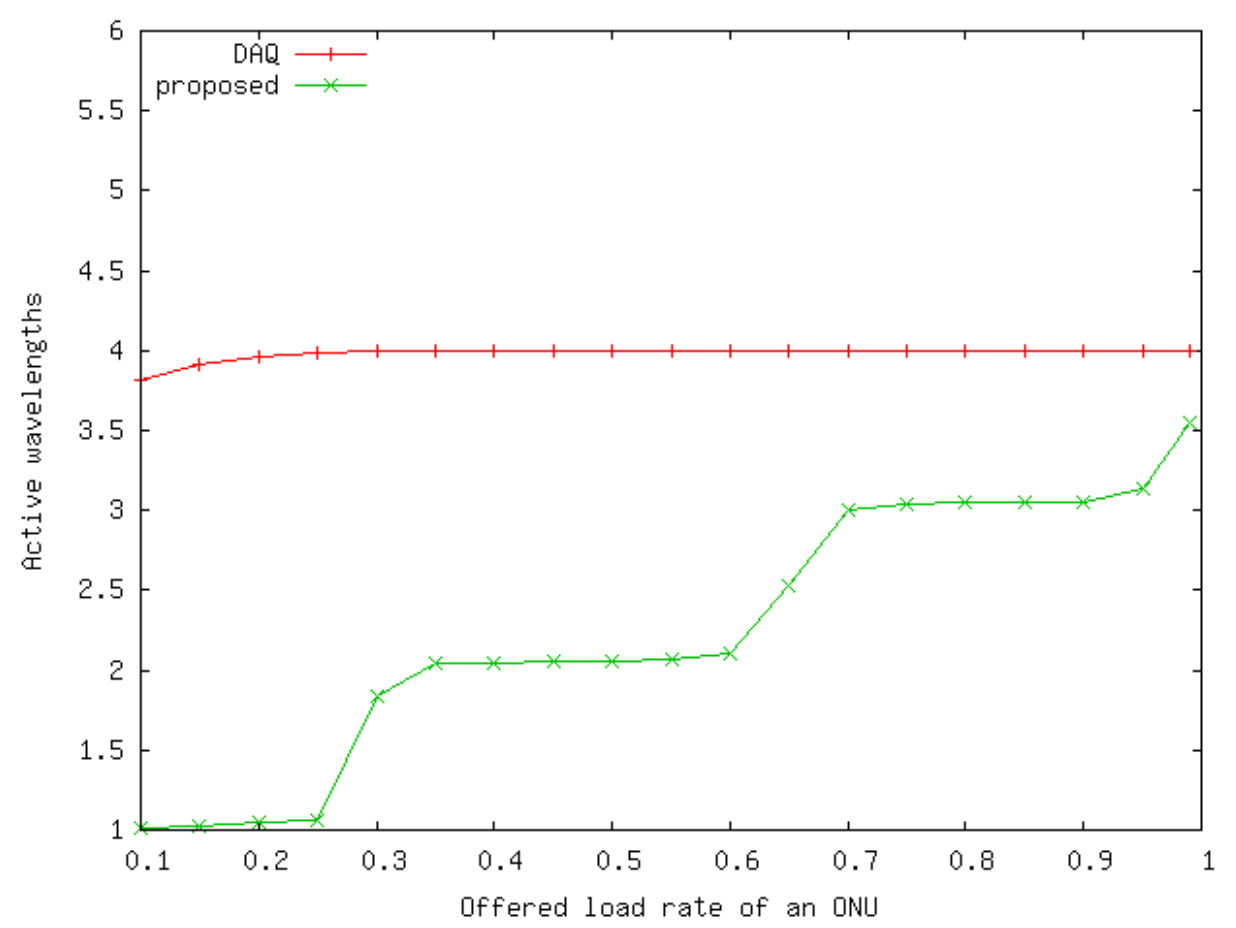

Figure 11. Average Number of Active Upstream Wavelengths

\section{Conclusions}

We introduce a new dynamic wavelength allocation method for a TWDM PON in which an ONU has a low-speed wavelength tuner which takes from few milliseconds to few seconds to change its wavelength. The OLT uses an average grant amount to infer the packet arrival rates of ONUs. If the average grant amount is less than the bandwidth sum of the upstream wavelengths, the number of active upstream wavelengths is decreased. Otherwise, the number of active upstream wavelengths is increased. Using computer simulations, we evaluate performance of the proposed method under self-similar traffic. Performance of the proposed method is comparable to that of the DAQ method in which the tuning time of an ONU is zero and multiple ONUs can simultaneously change their upstream wavelengths.

\section{References}

[1] ITU-T Rec. G.987.3 Rev.2, “10- Gigabit-capable passive optical networks (XG-PON): Transmission convergence (TC) specifications", (2010).

[2] ITU-T Rec. G.989.2 Draft, "40- Gigabit-capable passive optical networks (NG-PON2): Physical media dependent (PMD) layer specification", (2012).

[3] ITU-T Rec. G.987.1, "10 Gigabit-capable passive optical network (XG-PON): General requirements", (2010).

[4] M. S. Han, "Dynamic Wavelength Allocation for TWDM PON with Low-Cost ONUs", Asia-pacific Proceedings of Applied Science and Engineering for Better Human Life, vol.4, (2016), pp. 107-110

[5] M. S. Han, "Energy Efficient Dynamic Wavelength and Bandwidth Allocation for TWDM PON", Contemporary Engineering Sciences, vol. 7, no. 23, (2014), pp. 1303 - 1311

[6] ITU-T Rec. G.984.3, "40-Gigabit-capable passive optical networks (NG-PON2): Transmission convergence layer specification", (2015).

[7] M.S. Han, H. Yoo and D. S. Lee, "Development of Efficient Dynamic Bandwidth Allocation algorithm for XGPON", ETRI Journal, vol. 3, no. 1, (2013), pp. 18 - 26

[8] A. Dixit, B. Lannoo, D. Colle, M. Pickavert and P. Demeester, "Dynamic Bandwidth Allocation with Optimal Wavelength Switching in TWDM-PONs", 15th International Conference on Transparent Optical Networks (ICTON), (2013), pp. $1-4$.

[9] J. Zhang and N. Ansari, "Scheduling Hybrid WDM/TDM Passive Optical Networks With Nonzero Laser Tuning Time”, IEEE/ACM Transactions on Networking, vol. 19, no. 4, (2011), pp. 1014 - 1027. 
International Journal of Future Generation Communication and Networking Vol. 9, No. 11 (2016) 